\title{
Patrón de masticación según Ángulo Funcional de Planas y Prueba Funcional de Christensen y Radue en niños
}

\author{
Chewing pattern according to the Functional Angle of Planas \\ and the Christensen and Radue Functional Test in children
}

Fiorella Alexandra Torres Latorre ${ }^{1 a}$ Yury Miguel Tenorio Cahuana ${ }^{2 b}$
Dttps://orcid.org/0000-0002-8423-0107

isttps://orcid.org/0000-0001-8679-2050

Correspondencia: torreslatorre270494@gmail.com

\section{Resumen}

El presente artículo tuvo como objetivo establecer la concordancia del patrón de masticación según el Ángulo Funcional Masticatorio de Planas y la Prueba Funcional Masticatoria de Christensen y Radue en alumnos de 3 a 6 años de la I.E.I. N. ${ }^{\circ}$ 229-A Mafalda Céspedes Quelopana en Tacna en el 2019. La metodología del estudio fue descriptiva comparativa, de corte transversal y prospectiva. La población del estudio estuvo constituida por 56 alumnos; para ello se elaboraron dos fichas de recolección de datos: "Guía de observación del Ángulo Funcional Masticatorio de Planas" y "Guía de observación de la Prueba Funcional Masticatoria de Christensen y Radue". En los resultados con el Ângulo Funcional Masticatorio de Planas, en mujeres, se encontró masticación unilateral derecha con $41.94 \%$. Los resultados con la Prueba Funcional Masticatoria de Christensen y Radue, en varones, muestran masticación unilateral izquierda con $52.00 \%$. Conclusión: No existe concordancia del patrón de masticación según el Ángulo Funcional Masticatorio de Planas y la Prueba Funcional Masticatoria de Christensen y Radue y el índice de convergencia Kappa de Cohen es insignificante $(K=0.122)$.

Palabras clave: masticación bilateral, masticación unilateral, dentición decidua

\section{Abstract}

The objective of this article was to establish the concordance of the chewing pattern according to the masticatory functional angle of Planas and the Christensen and Radue masticatory functional test in students aged 3-6 years of the Kindergarten 229-A Mafalda Céspedes Quelopana of Tacna, 2019. The study methodology was comparative descriptive, cross-sectional and prospective. The study population consisted of 56 students. For this purpose, two data collection sheets were prepared: "Observation Guide for the Masticatory Functional Angle of Planas" and "Observation Guide for the Masticatory Functional Test of Christensen and Radue". In the results with the masticatory functional angle of planes in women, right unilateral mastication was found with $41.94 \%$. The results with the Christensen and Radue masticatory functional test, in men, show left unilateral mastication with $52 \%$. Conclusion: There is no concordance of the masticatory pattern according to the functional masticatory angle of Planas and the functional masticatory test of Christensen and Radue, and the Cohen's Kappa convergence index is insignificant $(K=0.122)$.

Keywords: bilateral mastication, unilateral mastication, deciduous dentition

\footnotetext{
${ }^{1}$ Investigador Independiente. Tacna, Perú

2 Universidad Nacional Jorge Basadre Grohmann. Escuela Profesional de Odontología. Tacna, Perú

a Cirujano Dentista

${ }^{\mathrm{b}}$ Especialista en Ortodoncia y Ortopedia Maxilar
} 


\section{Introducción}

La masticación es una actividad cíclica de retroalimentación sensorial controlada por un "generador de patrones" que se encuentra en el sistema nervioso central y que exhibe una variabilidad intra e interindividuos. ${ }^{1,2}$

Existen dos tipos de patrón de masticación: El patrón de masticación bilateral y el patrón de masticación unilateral. El patrón de masticación bilateral, conocido también como la masticación no inducida u orientada, típica, normal e instrumentada, consiste en alternar homogéneamente el lado de trabajo, en un número similar de veces, así mismo, la literatura define el patrón de masticación unilateral, cuando el número de ciclos masticatorios en un lado es aproximadamente $30 \%$ mayor que el número de ciclos masticatorios realizados en el lado opuesto. ${ }^{3}$

La masticación unilateral representa un riesgo por la pérdida de la audición, lo que no sucede con la masticación bilateral, lo cual resulta beneficioso para la salud del individuo. ${ }^{4}$

Para algunos autores, el dominio de la masticación unilateral ocurre con un $66 \%$ de ciclos masticatorios de un lado; en masticadores unilaterales crónicos ocurre con un $95 \%$. Así mismo, en estudios radiográficos se demuestra la alteración morfológica, específicamente del cuerpo mandibular, la rama ascendente y la cabeza del cóndilo mandibular. ${ }^{5}$

El dolor de una lesión cariosa, una restauración alta o una corona protésica mal confeccionada ocasiona una masticación unilateral. ${ }^{6}$ Hay una relación entre el dominio hemisférico y la preferencia por el lado de la masticación en la corteza sensoriomotora primaria responsable del apretamiento bilateral de los dientes. ${ }^{7}$

Hay cambios neurofisiológicos producidos por la masticación unilateral que afectan la función de la articulación témporo-mandibular y provocan trastornos acompañados de alteraciones de las funciones orofaciales. ${ }^{8}$ En relación a los lados de la masticación bilateral, no hay relevancia funcional; pero en la masticación unilateral, sea en el lado derecho o izquierdo en las cuales resalte el masetero dominante sobre el masetero subordinado, la diferencia se hallará en la capacidad de apretar y el manejo de la tensión. ${ }^{9}$

El Ángulo Funcional Masticatorio de Planas (AFMP) es un registro de la trayectoria mandibular durante las excursiones funcionales que determina el aumento de la dimensión vertical; representa la mínima dimensión vertical y se registra en el plano frontal, dichos ángulos deben ser iguales para ambos lados, lo que permite un proceso de masticación adecuado. ${ }^{10}$

La oclusión funcional es la que establece, entre las arcadas, el máximo de contactos intercuspídeos y cualquier movimiento excursivo producirá un aumento de la dimensión vertical del tercio inferior de la cara. Cuando esta posición no coincide con la oclusión céntrica, uno o ambos cóndilos se ubican fuera del fondo de la cavidad articular o comprimiendo alguna zona, y esto provoca lesiones agudas y crónicas de la articulación témporo-mandibular. Partiendo de una oclusión funcional, cuando la mandíbula realiza movimientos deslizantes que resbalen hacia ambos lados, lenta y relajadamente, se obtendrá, en recorrido inverso, los movimientos que la mandíbula realiza durante el acto masticatorio, los cuales siempre generarán un aumento de la dimensión vertical, aunque sea infinitamente pequeño. Planas (1996) registra estos movimientos en el plano frontal y los llama ángulos funcionales masticatorios; dichos ángulos permiten representar el desplazamiento centrífugo de la mandíbula reproduciendo la trayectoria de los dientes durante la masticación. ${ }^{10,11}$

Planas (1996), a través del ángulo funcional masticatorio, fundamenta que existe un mayor grado de masticación en el lado de menor angulación, el cual coincide con el lado de menor dimensión vertical, resultando una excelente herramienta de diagnóstico clínico. ${ }^{12}$ 


\section{Figura 1}

Registros del AFMP

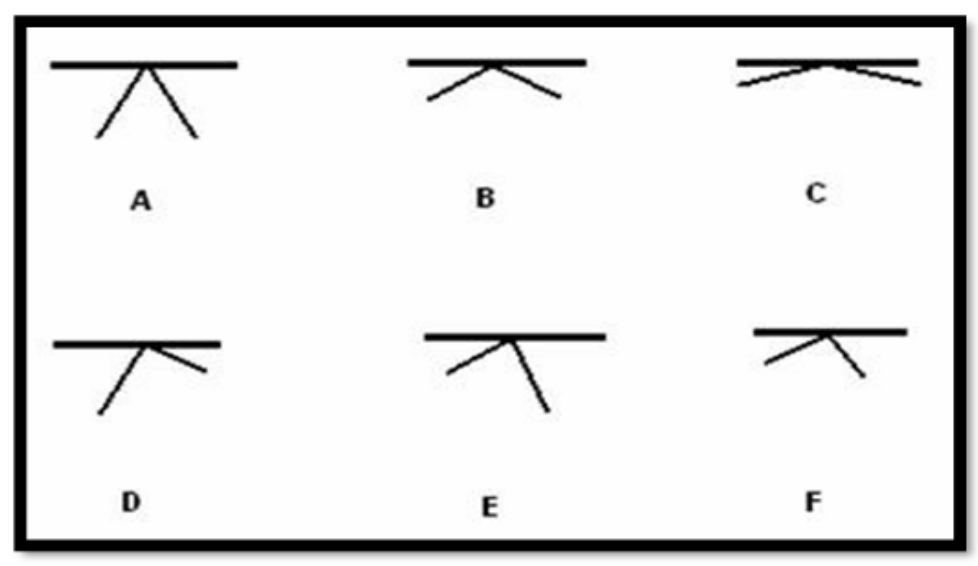

Nota. Tomado de la Revista Latinoamericana de Ortodoncia y Odontopediatría (2004).

Christensen y Radue (1985), con la Prueba Funcional Masticatoria, señalan que la preferencia del lado de masticación (CSP por sus siglas en inglés) ocurre cuando la masticación se realiza de manera exclusiva, consistente o predominante en un mismo lado. ${ }^{13}$

En 1985, Christensen y Radue determinan la preferencia del lado de masticación mediante un método de verificación visual directa a través de un bolo (goma de mascar).

A. Preferencia de lado de masticación consistente:

Derecha: $7 / 7$ golpes en el lado derecho.

Izquierda: $7 / 7$ golpes en el lado izquierdo.

B. Preferencia del lado de masticación predominante:

Derecha: $5 / 7$ o 6/7 golpes en el lado derecho.

Izquierda: 5/7 o 6/7 golpes en el lado izquierdo. ${ }^{14,15}$

A través del tratamiento en la cavidad oral se pretende mejorar o restablecer la función masticatoria, considerada como un componente importante en la salud oral. Es una de las funciones básicas del sistema estomatognático que puede afectar los hábitos dietéticos, la ingesta nutricional y, por consiguiente, influir en la salud del individuo en general. ${ }^{16-18}$

Este estudio tuvo como propósito determinar la concordancia del patrón de masticación según el Ángulo Funcional Masticatorio de Planas y la Prueba Funcional Masticatoria de Christensen y Radue en alumnos de 3 a 6 años para poder detectar tempranamente la implantación de una futura maloclusión.

\section{Análisis metodológico}

La población estudiada estuvo conformada por 56 niños de 3 a 6 años, a quienes se les aplicaron las fichas Guía de observación del Ángulo Funcional Masticatorio de Planas y la Prueba Funcional Masticatoria de Christensen y Radue.

Para la toma del Ángulo Funcional Masticatorio de Planas se pidió a los alumnos que se sienten en una silla, en un ambiente establecido. Se registró el ángulo utilizando hojas de transparencia sujetos en lentes sin lunas, indicando realizar lateralidades. 
Para la Prueba Funcional Masticatoria de Christensen y Radue se le proporcionó, al alumno, una goma de mascar sin azúcar (Trident de $450 \mathrm{mg}$ ), de un tamaño de $40 \times 10 \times 4 \mathrm{~mm}$ para que la mastique en un breve lapso de 15 segundos. Se pidió al alumno que deje de masticar y que realice la apertura bucal para observar el lado en el que estaba colocada la goma de mascar (ya sea a la derecha o a la izquierda). Este procedimiento se repitió después de intervalos de 15 segundos, cada uno durante siete veces.

Una vez recogidos los datos se realizó el control de calidad de fichas; posteriormente se elaboró una base de datos.

\section{Criterios de inclusión}

Alumnos que presenten dentición decidua completa.

Alumnos con dentición sana.

Alumnos voluntarios y colaboradores.

Alumnos matriculados en el año académico 2019.

\section{Criterios de exclusión}

Alumnos con tratamiento ortopédico.

\section{Resultados}

\section{Tabla 1}

Concordancia del patrón de masticación según el Ángulo Funcional Masticatorio de Planas y la Prueba Funcional Masticatoria de Christensen y Radue en niños

\begin{tabular}{|c|c|c|c|c|c|c|c|}
\hline & & & \multicolumn{4}{|c|}{$\begin{array}{c}\text { Prueba Funcional Masticatoria de } \\
\text { Christensen y Radue }\end{array}$} & \multirow{2}{*}{$\begin{array}{c}\text { Índice de } \\
\text { convergencia } \\
\text { Kappa de Cohen }\end{array}$} \\
\hline & & & Bilateral & $\begin{array}{l}\text { Unilateral } \\
\text { Derecho }\end{array}$ & $\begin{array}{l}\text { Unilateral } \\
\text { Izquierdo }\end{array}$ & Total & \\
\hline \multirow{8}{*}{ 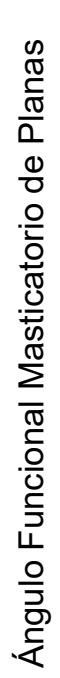 } & \multirow{2}{*}{ Bilateral } & $\mathrm{N} .^{\circ}$ & 4 & 5 & 8 & 17 & \multirow{8}{*}{0.122} \\
\hline & & $\%$ & 7.1 & 8.9 & 14.3 & 30.4 & \\
\hline & \multirow{2}{*}{$\begin{array}{l}\text { Unilateral } \\
\text { Derecha }\end{array}$} & $\mathrm{N} .^{\circ}$ & 3 & 9 & 11 & 23 & \\
\hline & & $\%$ & 5.4 & 16.1 & 19.6 & 41.1 & \\
\hline & \multirow{2}{*}{$\begin{array}{l}\text { Unilateral } \\
\text { Izquierda }\end{array}$} & $\mathrm{N} .^{\circ}$ & 2 & 4 & 10 & 16 & \\
\hline & & $\%$ & 3.6 & 7.1 & 17.9 & 28.6 & \\
\hline & \multirow{2}{*}{ Total } & $N .^{\circ}$ & 9 & 18 & 29 & 56 & \\
\hline & & $\%$ & 16.1 & 32.1 & 51.8 & 100 & \\
\hline
\end{tabular}

Nota. Fuente: Matriz de datos. 
Al efectuar el índice de convergencia Kappa de Cohen obtenemos un valor $\mathrm{K}=0.122$ (Insignificante), indicando que no existe concordancia del patrón de masticación según el Ángulo Funcional Masticatorio de Planas y la Prueba Funcional Masticatoria de Christensen y Radue.

Tabla 2

Patrón de masticación según el Ángulo Funcional Masticatorio de Planas, según sexo en niños

\begin{tabular}{|c|c|c|c|c|c|c|}
\hline \multirow{2}{*}{$\begin{array}{l}\text { Ángulo } \\
\text { Funcional } \\
\text { Masticatorio } \\
\text { de Planas }\end{array}$} & \multicolumn{2}{|c|}{ Mujeres } & \multicolumn{2}{|c|}{ Varones } & \multicolumn{2}{|c|}{ Total } \\
\hline & $\mathrm{N} .^{\circ}$ & $\%$ & $\mathrm{~N} .^{\circ}$ & $\%$ & $\mathrm{~N} .^{\circ}$ & $\%$ \\
\hline Bilateral & 12 & 38.71 & 5 & 20.00 & 17 & 30.36 \\
\hline $\begin{array}{l}\text { Unilateral } \\
\text { Derecha }\end{array}$ & 13 & 41.94 & 10 & 40.00 & 23 & 41.07 \\
\hline $\begin{array}{l}\text { Unilateral } \\
\text { Izquierda }\end{array}$ & 6 & 19.35 & 10 & 40.00 & 16 & 28.57 \\
\hline Total & 31 & 100.0 & 25 & 100.0 & 56 & 100.0 \\
\hline
\end{tabular}

Nota. Fuente: Matriz de datos.

La frecuencia del patrón de masticación mediante el Ángulo Funcional Masticatorio de Planas en mujeres es de $41.94 \%$ en la masticación unilateral derecha y en varones es de $40.00 \%$ en la masticación unilateral derecha e izquierda.

Tabla 3

Patrón de masticación mediante la Prueba Funcional Masticatoria de Christensen y Radue, según sexo en niños

\begin{tabular}{|c|c|c|c|c|c|c|}
\hline \multirow{2}{*}{$\begin{array}{l}\text { Prueba } \\
\text { Funcional } \\
\text { Masticatoria de } \\
\text { Christensen y } \\
\text { Radue }\end{array}$} & \multicolumn{2}{|c|}{ Mujeres } & \multicolumn{2}{|c|}{ Varones } & \multicolumn{2}{|c|}{ Total } \\
\hline & $\mathrm{N} .^{\circ}$ & $\%$ & $\mathrm{~N} .^{\circ}$ & $\%$ & $\mathrm{~N} .^{\circ}$ & $\%$ \\
\hline Bilateral & 6 & 19.35 & 3 & 12.00 & 9 & 16.07 \\
\hline $\begin{array}{l}\text { Unilateral Dere- } \\
\text { cha }\end{array}$ & 9 & 29.04 & 9 & 36.00 & 18 & 32.14 \\
\hline $\begin{array}{l}\text { Unilateral Iz- } \\
\text { quierda }\end{array}$ & 16 & 51.61 & 13 & 52.00 & 29 & 51.79 \\
\hline Total & 31 & 100.0 & 25 & 100.0 & 56 & 100.0 \\
\hline
\end{tabular}

Nota. Fuente: Matriz de datos. 
La frecuencia del patrón de masticación mediante la Prueba Funcional Masticatoria de Christensen y

Radue en la masticación unilateral izquierda es de $51.61 \%$ en mujeres y de $52.00 \%$ en varones.

\section{Discusión}

Se acepta la hipótesis nula que establece que no existe concordancia del tipo de masticación según el Ángulo Funcional Masticatorio de Planas y la Prueba Funcional Masticatoria de Christensen R. V. y Radue T en alumnos de 3 a 6 años en educación inicial. Esto podría deberse a que los individuos, normalmente, tragan los alimentos ingeridos después de 15 a 25 ciclos masticatorios en un tiempo de 15 a 25 segundos, así mismo podría deberse a la expresión de un comportamiento masticatorio motivacional.

La investigación determinó que el patrón de masticación mediante el Ángulo Funcional Masticatorio de Planas con mayor predominio es la masticación unilateral con un $69.64 \%$, que concuerda con las investigaciones de Arias JL, Ardon R., García F. y Linares V. Este resultado no concuerda con Travez G., en el cual predomina la masticación bilateral con un $67.00 \%$ del Ángulo Funcional Masticatorio de Planas.

Así mismo, se concluyó que el patrón de masticación unilateral del Ángulo Funcional Masticatorio de Planas con mayor frecuencia, según sexo, en los varones es de $80.00 \%$, esto concuerda con la investigación de Arias J. L. Por otro lado, los resultados no concuerdan con Travez G., en los cuales se determinó que el patrón de masticación unilateral con mayor predominio, según sexo, en las mujeres es de $61.22 \%$, mediante el Ángulo Funcional Masticatorio de Planas.

Los resultados obtenidos del patrón de masticación unilateral, en mujeres, con mayor predominio, mediante el Ángulo Funcional Masticatorio de Planas, es el derecho con un $68.42 \%$, que concuerda con los resultados obtenidos de Travez G., Arias JL, Ardon R., García F. y Linares V. Por otro lado, se determinó que el patrón de masticación mediante la Prueba Funcional Masticatoria de Christensen y Radue con mayor predominio es la masticación unilateral con un $83.93 \%$, que concuerda con las investigaciones de Travez G., Arias JL, Ardon R., García F. y Linares V.

Finalmente, se resolvió que la masticación unilateral mediante la Prueba Funcional Masticatoria de Christensen y Radue, con mayor predominio según sexo, en los varones, es de $88.00 \%$, concordante con la investigación de Arias JL. Así mismo, es discordante con la investigación de Travez G., en la cual se determinó que la masticación unilateral con mayor predominio según sexo, en las mujeres, es de $61.22 \%$, mediante la Prueba Funcional Masticatoria de Christensen y Radue.

\section{Conclusiones}

No existe concordancia del patrón de masticación según el Ángulo Funcional Masticatorio de Planas y la Prueba Funcional Masticatoria de Christensen y Radue en alumnos de 3 a 6 años; ya que el índice de convergencia Kappa de Cohen es insignificante $(K=0.122)$.

La frecuencia del patrón de masticación según el Ángulo Funcional Masticatorio de Planas según sexo en mujeres es de $41.94 \%$ para la masticación unilateral derecha y de $40.00 \%$ para la masticación unilateral derecha e izquierda en varones, dando un total, con predominio de masticación unilateral derecha, de $41.07 \%$.

La frecuencia del patrón de masticación mediante Christensen y Radue para la masticación unilateral izquierda es de $51.61 \%$ en mujeres y de $52.00 \%$ en varones.

\section{Recomendaciones}

Implementar el Análisis del Ángulo Funcional Masticatorio de Planas en las historias clínicas dentales para denotar la presencia del patrón de masticación unilateral, propiciando un tratamiento interceptivo.

Implementar estudios del análisis del Ángulo Funcional Masticatorio de Planas en niños de 3 a 6 años para obtener datos epidemiológicos actualizados. 


\section{Referencias}

1. Bourdiol P, Mioche L. Correlations between functional and occlusal tooth-surface areas and food texture during natural chewing sequences in humans. Archives of Oral Biology. 2000 August; 45(8):691-9.

2. Flores E. Evaluación de la fiabilidad y validez de métodos que determinan el lado de preferencia masticatorio. Tesis doctoral. Universidad de Barcelona]. Repositorio Digital de la UB. 2014.

3. Hovsepian M. Algunos aspectos clínicos sobre la masticación unilateral. Revista Latinoamericana de Ortodoncia y Odontopediatría. 2017.

4. Ran Lee, Y. R., Choi, J. S., \& Kim, H. E. (2018). Unilateral Chewing as a Risk Factor for Hearing Loss: Association between Chewing Habits and Hearing Acuity. The Tohoku journal of experimental medicine. 2018. 246(1), 45-50.

5. Simoes W. Ortopedia funcional de los maxilares a través de la rehabilitación neurooclusal 3ra edición. Editorial artes médicas Sao Paulo - Brasil. 2003. Vol. I. pág.116

6. Planas P. Rehabilitación Neurooclusal (RNO). 2da edición. Editorial Amolca. Madrid - España.1996.

7. Jiang H., Liu H., Liu G., Jin Z., \& Liu X. The effects of chewing-side preference on human brain activity during tooth clenching: an fMRI study. Journal of oral rehabilitation. 2010. 37(12), 877-883.

8. La Touche, R., Losana-Ferrer, A., Pascual-Vaquerizo, E., Suso-Martí, L., Paris-Alemany, A., ChamorroSánchez, J., \& Cuenca-Martínez, F. Orofacial sensorimotor behaviour in unilateral chewing: Acomparative analysis in asymptomatic population. Physiology \& behavior. 2019; 212, 112718.

9. Busato, A., Balconi, G., Vismara, V., Bertelè, L., Garo, G., \& DE Gregorio, D. (2017). Analysis of masseter deformation patterns during a maximum exertion clenching in patients with unilateral chewing. ORAL \& implantology, 9(Suppl 1/2016 to N 4/2016). 2016; 54-64.

10. Urquizo MdR. Detección precoz de anomalías funcionales masticatorias a través del análisis del ángulo funcional masticatorio de planas en niños con dentición mixta en las instituciones educativas del distrito Gregorio Albarracín Lanchipa 2018. Tesis de Pregrado. Tacna: Universidad Nacional Jorge Basadre Grohmann, Facultad de Odontología; 2018.

11. Mamani K. Anomalías funcionales masticatorias y su relación con trastornos temporomandibulares en niños de 9 a 10 años de edad - Institución Educativa Enrique Paillardelle Tacna, 2018. Tesis de Pregrado. Tacna: Universidad Nacional Jorge Basadre Grohmann, Escuela de Odontología; 2019.

12. Álvarez B. Filosofía de Pedro Planas aplicada al diagnóstico y tratamiento en ortodoncia dento maxilo facial. Revista electrónica del Instituto Universitario Centro de Estudio y Diagnóstico de las Disgnacias de Uruguay. 2017.

13. Christensen RV, Radue T. Lateral preference in mastication: a feasibility study. Journal of Oral Rehabilitation. 1985; 12(421-427).

14. Nayak UA, Sharma R, Kashyap N, Prajapati, Kappadi D, Wadhwa S, vet al. Association between Chewing Side Preference and Dental vCaries among Deciduous, Mixed and Permanent Dentition. Journal of vClinical and Diagnostic Research. 2016 September; 10(9).

15. Marchesan I. Fundamentos de Fonoaudiología S.A GR, editor. Sao Paulo: Editorial Médica Panamericana; 2002.

16. Flores O. Evaluación de la fiabilidad y validez de métodos que determinan el lado de preferencia masticatorio. Tesis doctoral. Barcelona: Universidad de Barcelona, Ciencias Odontológicas; 2016.

17. N'Gom, DDS I, DDS, Ph AW. Influence of impaired mastication on nutrition. Journal Prosthetic Dentistry. 2002 June; 87(6):667-73.

18. Baba K., John, M., Inukai, M., Aridome, K., Igarahsi, Y. Validating an alternate version of the chewing function questionnaire in partially dentate patients. BMC oral health, 9, 9; 2009.

- Conflicto de intereses: La presente investigación no presenta conflicto de intereses entre los investigadores.

- Fuente de financiamiento: La presente investigación fue financiada por los investigadores. 